\title{
25
}

\section{Performance Space of a GI/G/1 Queueing System Under a Percentile Goal Criterion}

\author{
Nitin Agarwal and Ioannis Viniotis, \\ Center for Communications and Signal Processing, \\ Department of Electrical and Computer Engineering, \\ North Carolina State University, Raleigh, N.C. 27695-7914, U.S.A. \\ e-mail: candice@ececho.ncsu.edu.
}

The performance of a preemptive priority queueing system with a single server and multiple customer classes is studied. We assume independent identically distributed general interarrival and general service times for each class of customers. The performance of a customer class is characterized by a prespecified waiting time objective and the goal is to guarantee that a given fraction, or percentile, of the customers from the class will be served with their waiting times less than the specified waiting time objective. We define this as the percentile goal criterion. In this paper, we characterize the performance space of such 2 class and 3 class queueing systems with single percentile goal.

\section{Introduction}

A key problem in computer networks is to deliver data from source to destination with guarantees on the waiting times experienced by data within the network. A common approach to solve this problem is to split the waiting time guarantees from source to destination into the guarantees at nodes along the way and then provide guarantees on each node. In this way, a multiple node problem can be converted into a single node problem. A simple procedure, which is shown to work well for small losses, is to split the overall guarantees into guarantees at individual nodes evenly across all nodes [9].

Priority queueing models can be successfully used to analyze and control the performance of such nodes in computer networks in which different classes of data compete for a link with limited capacity. A multiclass preemptive priority queueing system is one that has multiple data types which differ in their arrival processes, service processes and performance requirements and the data packets belonging to the class with higher priority have preemptive priority. We model our problem as a multiclass preemptive priority queueing problem.

In this paper, we study the performance characterized by a prespecified waiting time objective and the goal is bo guarantee that a given fraction $X_{i j}$ of packets of class $i$ will be served with their waiting times less than the specified waiting time objective $g_{i j}$ where $j=1,2, \ldots, M$, for some integer $M$. Clearly, for large $M$ and small $g_{i, j+1}-g_{i j}$, this criterion reduces to the guarantee that $X_{i j}$ fraction of packets will have waiting time $g_{i, j+1}$ which amounts to giving guarantees on the actual waiting times. We define this, 
loosely, as the percentile goal criterion. The percentile goal criterion is formally defined on page 3. This criterion becomes important in the case of, for example, voice traffic where a good performance in terms of mean delay is not sufficient to ensure good output voice quality. Here, what is also important is a good performance in terms of say, the 99th percentile of delay. This allows the destination to select the target playout delay appropriately for incoming packets, with the assurance that packet loss is within tolerable limits [6]

For a given system (i.e., the critical resources, the arrival processes of the classes, their service processes and $g_{i j}$ 's), percentile goal criterion can not be met for all prespecified $X$ 's where $X=\left[X_{i, j}\right]$. The set of values of $X$ 's for which the percentile goal criterion can be met is called the performance space. In general, the size and shape of the performance space will be dependent upon a given system and the class of scheduling policies allowed. An interesting problem is to characterize the performance space for a given system. For average waiting time criteria, the performance space (i.e., the set of achievable waiting times) is characterized by the conservation laws for expected waiting times [5, ?]. For this reason, characterization of performance space for percentile goal criterion can also be viewed as a first step in an attempt to develop the conservation laws for actual waiting times.

In this paper, we characterize the performance space for 2 class and 3 class systems with single percentile goals through a series of scheduling algorithms which achieve the corner points of the performance space for the given system under work-conserving scheduling policies. We are currently working on generalizing these results to $N$ classes and $M$ percentiles.

Rest of the paper is organized in the following way:

In section 2 , we describe the queueing model. In section 3 , we formulate the problem and in section 4 , the set of admissible policies is described. In section 5 , we present the optimal algorithms. In section 6 , we describe the performance space for a 2 classes and a single percentile goal system. In section 7, we extend the results to 3 classes and a single percentile goal system. This extension is not trivial for the reasons described later. All the results are stated without proof. The interested reader can refer to [1] for details of proof.

\section{The Queueing Model}

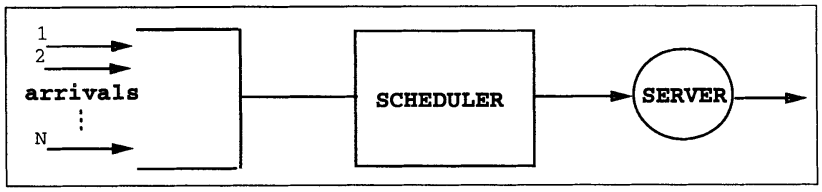

Figure 1. The single server system with 2 classes 
Consider the system of Figure 1.

Here, we have a single server queue with $N$ classes of customers. We assume a stable system with iid interarrival and service times for customers in a class and service times are independent of the arrival process. The customers which belong to class $i$ arrive at the system according to a general process, $A_{i}($.$) . The service times follow a general$ distribution $B_{i}($.$) for class i$.

\section{The General Percentile Goal Criterion Problem}

Let $X_{i j}$ denote the percent of class $i$ packets that can tolerate an actual waiting time of up to $g_{i j}$ time units. Let $W_{i}$ denote the actual waiting time for packets of class $i$. Then the percentile goal criterion problem can be expressed mathematically as follows:

Find an algorithm that guarantees that for all classes $i=1,2, \ldots, N$, we have:

$\begin{array}{rlr}W_{i} \leq g_{i 1} & \text { for } X_{i 1} \text { fraction of packets } \\ g_{i 1}<W_{i} \leq g_{i 2} & \text { for } X_{i 2} \text { fraction of packets, } \\ \vdots & \\ g_{i, M-1}<W_{i} \leq g_{i M} & \text { for } X_{i M} \text { fraction of packets, }\end{array}$

where, of course, $X_{i 1}+\ldots+X_{i M}=1$ and $g_{i j}$ 's are constants for all the values of $i$ and $j$ and $g_{i M}$ could possibly be infinity.

The above formulation can be used to specify performance requirements on the actual waiting times. So, for example, we have a link on which 3 classes of packets need to be scheduled. The 3 classes are data, voice and video respectively denoted as class 1,2 and 3 . The class 1 packets are not very delay sensitive and they can tolerate a large delay of $10 \mathrm{~s}$. The class 2 packets are delay sensitive and say, $95 \%$ of them should not experience a delay of more than $25 \mathrm{~ms}$. The class 3 packets are not that sensitive to delay as they are to the variation in delay. So, we can have a relatively large delay of 200 ms for $95 \%$ of them. This problem can be formulated in our framework as follows:

$$
\begin{array}{ll}
W_{1} \leq 10 & \text { for } 100 \% \text { packets } \\
W_{2} \leq .025 & \text { for } 95 \% \text { packets, } \\
W_{2}>.025 & \text { for } 5 \% \text { packets, } \\
W_{3} \leq .2 & \text { for } 95 \% \text { packets } \\
W_{3}>.2 & \text { for } 5 \% \text { packets }
\end{array}
$$

In practice, a fraction of packets receiving very large delays can be tolerated as illustrated in the above example.

As we increase $M$, the percentile goal criterion can be thought of as an attempt to shape the distribution function of the waiting time Figure 2. In this sense, it is a more general criterion than the maximum waiting time criteria or maximum variance of waiting time criteria where both problems can be solved once the distribution function of the waiting time is known.

As a first step towards solving the general problem, we will be focusing on single percentile goal problems i.e., $M=1$. 


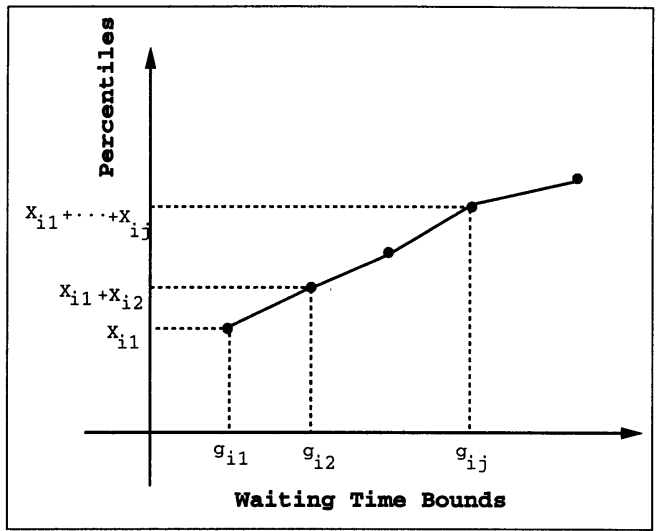

Figure 2. The percentile goal criteria as distribution function

\section{The Admissible Policies}

In this paper, we will be discussing the percentiles obtained when a particular scheduling policy $f$ is followed in scheduling different jobs from various classes on the server. Since the analysis for any policy $f$ can be extremely tedious, we will be restricting ourselves to a fairly general set of scheduling policies called work conserving policies[4]. Also, for the reasons described earlier, we have assumed that we will be considering only preemptive priority disciplines. Let $\Gamma_{1}$ denote the set of such scheduling rules. Henceforth, unless we specify, all the policies we consider here are assumed to be in the set $\Gamma_{1}$.

Expected waiting time of a class is independent of which job from that class is actually served so long as the server serves some job which needs service. But for actual waiting times, not only that we have to specify which class is going to be served, we also need to specify which job from that class is going to be served by our scheduling policy. Thus, a complete scheduling policy $f$ which guarantees a given percentile goal will have 2 independent components: namely, a between classes scheduling policy and a within class scheduling policy.

The first component specifies the priority order for the classes at time $t$ for all $t$. This affords us an opportunity to alter the percentiles by changing the priorities of the customer classes. After the class of job which will receive service is determined by the first component of the scheduling policy, the second component specifies which job from that class will receive service. Here, we can specify different scheduling policy for each class. Typical examples of a within class scheduling rules would be First In First Out(FIFO), Last In First Out(LIFO) etc. In general, following different within class scheduling policies for the same class $i$ will lead to different percentiles $X_{i}$. In the next section, we will be describing the within class scheduling policies which will give the optimal (i.e., the maximum and the minimum) percentiles for the class $i$.

Let $p$ be the scheduling policy for scheduling in between classes and $q_{i}$ be the within 
class scheduling policy for class $i$. Then, for $N=2$, the triplet $\left(p, q_{1}, q_{2}\right)$ will describe the scheduling policy completely at any time $t$. We will denote such scheduling policy by $f$. It should be noted that $p$ and $q_{i}$ 's are all functions of time $t$. Thus, the policy $f$ is also a function of time $t$. If $f$ is same at all $t$, then it is a static scheduling policy. Otherwise, in general, $f$ is a dynamic scheduling policy. In this paper, we will be concerning ourselves with both kind of policies.

Now we define the scheduling policies considered in this paper more closely. By $f \in \Gamma_{1}$, we mean that $q_{1}, q_{2}$ and $p$ are $\in \Gamma_{1}$ and the class which has priority at a given instant $t$ has preemptive priority over the other class. Within class preemptions are not allowed. Also, preemptive priorities can be changed only at the beginning of a busy period and they will remain same throughout that busy period.

Now, having described the rules of the game i.e., the queueing system and the set of the scheduling policies, we want to describe the set of percentiles $X$ achievable for a given arrival processes $A_{i}($.$) and the service processes B_{i}($.$) forall i=1, \ldots, N$ by varying the scheduling policies $f \in \Gamma_{1}$. This we intend to achieve by describing the policies $f$ which will achieve the maximum and the minimum percentiles. As we noted earlier, the policy $f$ has 2 components. It is easy to see that, given same arrival and service processes and same within class scheduling disciplines, the maximum percentile for a class will be achieved when it has the highest priority among all the classes and the minimum percentile will be obtained when it has the lowest priority. Thus, the first component of the optimal policies $f$ is easy to fix. The second component is not so obvious. This we describe now in the next section.

\section{Within Class Optimal Scheduling Policies}

In the last sections, we described the model and the set of admissible policies. Now, we will present within class algorithms which will achieve the optimal performances i.e., the maximum and the minimum percentiles, for that class. In the next sections, we will use these algorithms to describe the performance space of the GI/G/1 system under percentile goal criterion. Here, we will fix $N=2$ for convenience in analysis. The results of this section can be easily extended to a general case. Here, our main results are Theorems 1 and 2. We prove these theorems through a series of lemmas. All the proofs are given in [1].

Let $N_{i}(t)$ be the total number of jobs of class $i$ which arrive into the system and $Y_{i}(f, t)$ be the number of jobs of class $i$ which were served with waiting time less than or equal to $g_{i}$ in the interval $[0, \mathrm{t})$. Let $N_{i}^{k}=$ and $Y_{i}^{k}(f)$ be the corresponding quantities for the $k^{\text {th }}$ busy period.

Then, $X_{i}(f)$, the steady state class $i$ percentile obtained when policy $f$ is followed is defined as follows:

$X_{i}(f) \triangleq \lim _{t \rightarrow \infty} \frac{Y_{i}(f, t)}{N_{i}(t)}=\lim _{k \rightarrow \infty} \frac{\sum_{j=1}^{k} Y_{i}^{j}(f)}{\sum_{j=1}^{k} N_{i}(j)}$

As we can have work conserving policies which are dynamic and are such that the value of $Y_{i}^{k}(f)$ oscillates from one busy period to another, the above limit may not exist for all $f \in \Gamma_{1}$. 


\section{1. $g_{i}$-FIFO Policy}

This policy maximizes the percentiles obtained for a given class. It is defined as follows:

Let us assume that we have to choose a class $i$ job at this instant for the server to serve. This can happen either because class $i$ has the highest priority of all the jobs waiting or class $i$ has low priority but no job of higher priority is waiting.

Among all the jobs waiting to receive service of class $i$, choose the one with the maximum waiting time upto $g_{i}$. Only when all the jobs with waiting time less than or equal to $g_{i}$ are exhausted, we may choose a job with waiting time higher than $g_{i}$. Notice that the policy does not specify which particular job with waiting time higher than $g_{i}$ should be chosen.

Thus, this policy chooses jobs from class $i$ according to FIFO if their waiting times are less than the cut-off of $g_{i}$. Once a job has already waited for more than $g_{i}$ time units, it's contribution towards the percentile goal becomes zero and then the policy tries to schedule all such jobs as late as possible. Now, we will establish the optimality of this intuitively appealing approach.

Let $f^{\prime}$ denote the policy when class 1 had preemptive priority and $g_{i}$-FIFO was the within class policy followed for all classes. Following the notation of page 4 , let us denote this policy by $f^{\prime}=\left(p^{\prime}, q^{\prime}, r^{\prime}\right)$. Thus, under $f^{\prime}$, we have $q^{\prime}$ and $r^{\prime}$ as the within class policies for class 1 and 2 i.e.,

$q^{\prime} \equiv g_{1}$-FIFO and $r^{\prime} \equiv g_{2}$-FIFO. Also, $p^{\prime} \equiv(1,2)$ where $(1,2)$ denotes that class 1 has preemptive priority over class 2 . Note that $f^{\prime}$ is a static policy in the sense we discussed in the last section.

The following lemma shows that the percentiles exist for the policy $f^{\prime}$ we described above.

Lemma $1 X_{i}\left(f^{\prime}\right)$ exists.

The following theorem establishes the optimality of the policy $f^{\prime}$.

Theorem $1 X_{i}\left(f^{\prime}\right)$ is the maximum obtainable percentile of class $i$ for all $f \in \Gamma_{1}$ such that the limit $X_{i}(f)$ exists for given $g_{i}$ 's. That is,

$X_{i}(f) \leq X_{i}\left(f^{\prime}\right) \forall f \in \Gamma_{1}$

where $f$ is such that $X_{i}(f)$ exists.

\section{2. $g_{i}$-LIFO Policy}

This policy minimizes the percentiles obtained for a class. The minimum percentile obtained is not zero because we are following a work conserving system and in general, there will always be some jobs of all classes which will receive service after waiting for zero unit of time. Although, normally we will not be interested in applying this policy, it is neverthless useful in giving a complete description of the performance space.

A $g_{i}$-LIFO policy (denoted by $q_{i}^{\prime \prime}$ ) is described as follows: 
Of all the class $i$ jobs waiting, choose any job $n$ with $W_{i}(n)>g_{i}$. If there is no such job, then choose the job with the minimum waiting time.

Here, the scheduler is making the best possible efforts within the constraint of work conserving rules to not serve the jobs which will contribute to the percentile goal. First, it serves jobs which have already waited for longer than $g_{i}$ and then, it chooses the job which has spent minimum time in the system with the hope that while this job is being served, the other jobs will become "useless". This intuition can be established mathematically in the same way as the Theorem 1 and then we have the following theorem.

Let $f^{\prime \prime}=\left(p, q_{1}^{\prime \prime}, q_{2}^{\prime \prime}\right)$, where $\mathrm{p}$ is any between class policy. As before, we are varying only $q_{i}$, the within class policy for some class $\mathrm{i}$, say $i=1$, for the next theorem.

Theorem $2 X_{1}\left(f^{\prime \prime}\right) \leq X_{1}(f) \forall f \in \Gamma_{1}$ such that $f=\left(p, q_{1}, q_{2}^{\prime \prime}\right)$ and $X_{1}(f)$ exists.

Thus, we have shown that $g_{i}$-FIFO and $g_{i}$-LIFO within class scheduling policies respectively maximize and minimize the percentiles of class $i$. Thus, for a 2 class problem, the percentile of class $i$ is maximized when it has the highest priority throughout all the busy periods and $g_{i}$-FIFO was followed as the within class policy. Similarly, the percentile of class $i$ is minimized when it has the lowest priority throughout all the busy periods and $g_{i}$-LIFO was followed as the within class policy. In the next section, we use this and similar results to construct the performance space of a 2 class, single percentile system.

\section{The Performance Space of 2 Class System}

In this section, we want to focus on the following problem:

Let $W_{i}(f)$ denote the waiting time of a job of class $i$ under a scheduling policy $f$ and let we want that $X_{i}$ percentiles of the jobs of class i have waiting times less than or equal to $g_{i}$ for $\mathrm{i}=1$ and 2. Let $\mathrm{X}=\left(X_{1}, X_{2}\right)$ be the point with coordinates as $X_{1}$ and $X_{2}$. For a given values of $g_{1}$ and $g_{2}$, we want to find all achievable $\mathrm{X}$ 's for the given GI/G/1 system.

Now, we have the following lemma which is important because it tells us that the performance space is convex.

Lemma 2 For a fixed value of $g_{1}$ and $g_{2}$, if points $X^{1}$ and $X^{2}$ are achievable, then all points on the line segment joining $X^{1}$ and $X^{2}$ are achievable. This also implies that the performance space is convex.

In the following, we show that the performance region is as shown in the Figure 3. Here, point $\mathrm{A}$ is obtained by the following algorithm:

The server will serve both classes according to $g_{i}$-FIFO within class policy. In almost all the busy periods, class 1 will have higher priority than class 2 . The point $\mathrm{B}$ is obtained similarly by reversing the roles of classes 1 and 2 .

The point $\mathrm{E}$ is obtained in the following way:

The server, now, will serve class 1 jobs according to $g_{i}$-FIFO and class 2 jobs according to $g_{i}$-LIFO and class 1 jobs have priority over class 2 jobs in all busy periods. The point $\mathrm{F}$ is obtained by reversing the roles of classes 1 and 2 . 


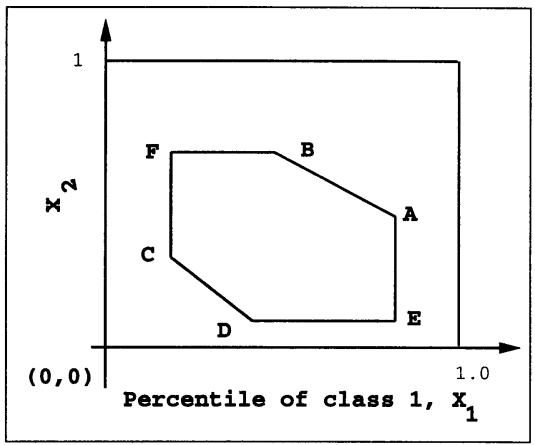

Figure 3. The performance region for 2 class system

The point $\mathrm{D}$ is obtained in the following way:

The server will serve both classes according to $g_{i}$-LIFO within class policy. In all the busy periods but a finite number of them, class 1 will have higher priority than class 2 . The point $\mathrm{C}$ is obtained similarly by reversing the roles of classes 1 and 2 .

Lemma $3 A B$ is the separating hyperplane of the performance space i.e., all points on one side of $A B$ are not achievable by any policy $f \in \Gamma_{1}$. Also, it denotes the maximal performance as the points $A$ and $B$ denote the maximum achievable percentiles for classes 1 and 2 respectively.

Thus, the line $\mathrm{AB}$ gives a bound on the upper performance region. Similarly, we have the following theorem which bounds the lower performance region.

Lemma $4 C D$ is the separating hyperplane of the performance space i.e., all points on one side of $C D$ are not achievable by any policy $f \in \Gamma_{1}$. Also, it denotes the minimal performance as the points $C$ and $D$ denote the minimum achievable percentiles for classes 1 and 2 respectively.

Now, we have the desired performance space.

Theorem 3 For all policies $f \in \Gamma_{1}$, the region ABFCDE, as shown in Figure 3, represents the set of all achievable points.

Thus, ABFCDE represents the desired performance space. Normally, a user will be interested only in the line $\mathrm{AB}$ of the performance region as it represents the space of maximum performance. But, sometimes when some other policy like FIFO etc. is being followed, then the performance will lie in the interior of the region ABFCDE. This kind of performance region can be used to make call admission control decisions. For example, if a user wants to have performance which lies outside this region, he can be denied service immediately. Another usefulness of this region lies in the fact that sometimes, depending 
on the arrival processes, service processes and the $g_{i}$ 's, the lines $\mathrm{AB}$ and $\mathrm{CD}$ may be too close to each other. In that case, we can follow any easily implementable within class policy without loosing much on the performance.

The above performance space can be described by the following set of inequalities. They can mean to interpret that a point $\left(X_{1}, X_{2}\right)$ is achievable if it satisfies following inequalities:

$0 \leq X_{i} \leq 1 \forall i=1,2$.

$\left(X_{2 B}-X_{2 A}\right)\left(X_{1}\right)+\left(X_{1 A}-X_{1 B}\right)\left(X_{2}\right) \leq\left(X_{1 A}-X_{1 B}\right)\left(X_{2 A}\right)-\left(X_{2 B}-X_{2 A}\right)\left(X_{1 A}\right)$

$\left(X_{2 C}-X_{2 D}\right)\left(X_{1}\right)+\left(X_{1 D}-X_{1 C}\right)\left(X_{2}\right) \geq\left(X_{1 D}-X_{1 C}\right)\left(X_{2 D}\right)-\left(X_{2 C}-X_{2 D}\right)\left(X_{1 D}\right)$

$X_{1} \leq X_{1 A}, X_{1} \geq X_{1 C}, X_{2} \leq X_{2 B}, X_{2} \geq X_{2 D}$

We also have the following equalities:

$X_{2 D}=X_{2 E}, X_{2 B}=X_{2 F}, X_{1 A}=X_{1 E}, X_{1 F}=X_{1 C}$

For a 2 class problem, we get only 2 points when we change the priorities and keep the within class policies fixed at $g_{i}$-FIFO for both classes. These 2 points trivially lie on a 2-D hyperplane. For a 3 class problem, we will get 6 points, one each for the 6 priority vectors and there is no guarantee that these 6 points will lie on a 3-D hyperplane. In the next section, we show that they all lie on a plane and characterize the performance space for 3 classes and single percentile goal problem.

\section{The Performance Space of 3 Class System}

The scheduling policies $f$ in this section, will be 4 -tuples with 3 member for the within class scheduling policies for 3 classes and the $4^{\text {th }}$ member for the priority vector $\mathrm{p}$ among them. Here, $\mathrm{p}$ will be a vector with 3 elements.

Same within class policies as for $N=2$ will still be optimal. The performance region for $N=3$ is shown in the Figure 4 .

Thus, for 3 classes, we will have the performance region as 3 dimensional polyhedron formed by removing the opposite corners of a polyhydroid by 3-D planes. The length of the polyhydroid along the $i^{t h}$ axis is equal to range of performance achievable for the $i^{\text {th }}$ class. The performance is maximum, i.e. the percentiles of job with waiting time less than or equal to $g_{i}$ is maximum, when the class $i$ has the highest priority and the within class policy for the class $i$ is $g_{i}$-FIFO. The performance is minimum when the class $i$ has the lowest priority and the within class policy for the class $i$ is $g_{i}$-LIFO. These edges of polyhydroid will be parallel to the corresponding axis. The corners of the polyhydroid corresponding to the maximum and the minimum percentiles of all classes will not be achievable because that implies giving the highest or the lowest priority to all the classes at the same time. Instead, as we will show, these corners will be limited by 3-D hyperplanes.

There will be 3 ! points, one for each priority order, obtained by following $g_{i}$-FIFO policy for all 3 classes. We generalize Lemma 3 of the previous chapter and show that all 6 points thus obtained will have to lie on a 3-D hyperplane. 


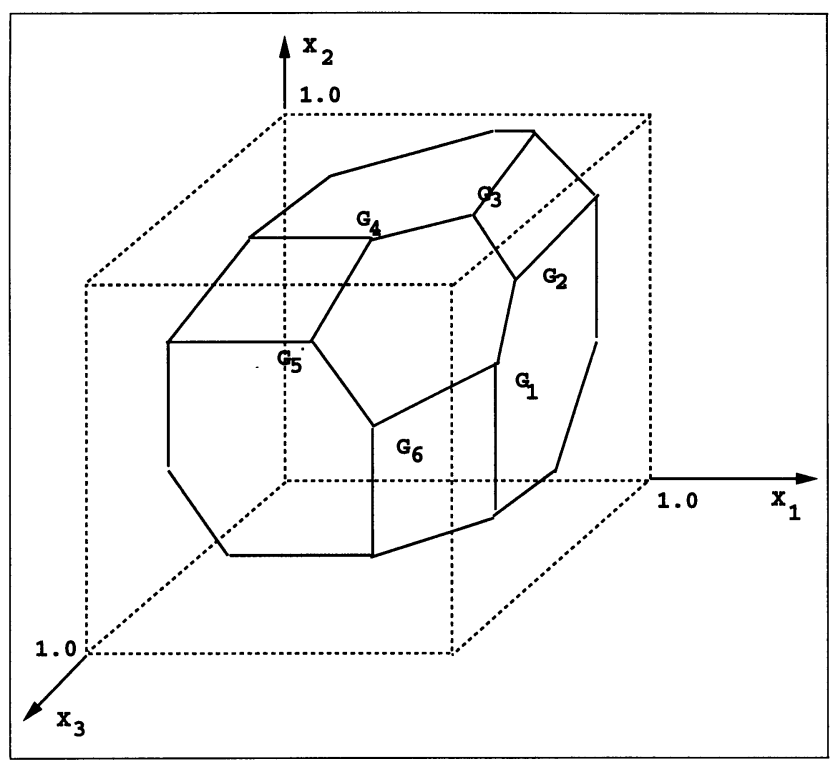

Figure 4. The Performance Space of a 3 Class System

Lemma 5 If within class policies for all the classes are fixed as $g_{i}-F I F O$, then the percentile of a class can not be increased without decreasing the percentile of some other class.

Lemma 6 All 3! points obtained by following $g_{i}$-FIFO policy for all the classes lie on an 3-D hyperplane and no point above this hyperplane is achievable.

Lemma 7 All 3! points obtained by following $g_{i}$-LIFO policy for all the classes lie on an 3-D hyperplane and no point below this hyperplane is achievable.

Theorem 4 The region shown in the Figure 4 is the performance space for $n=3$ for all policies $f \in \Gamma_{1}$.

\section{Conclusion}

In this paper, we have described and proved algorithms which give us the optimal percentile performances achievable among all the policies within the class of preemptive, work-conserving policies. Also, these algorithms enabled us to characterize the performance space of the single server, multiple class queueing system, under percentile goal criteria for 2 and 3 classes. Here, we have shown that the performance space is convex and we have described the algorithms to achieve the corner points of this space. Any other point can be obtained, at least theoretically, by following a suitable randomizing policy. In future works, we intend to generalize these results to $N$ classes and $M$ percentiles. 


\section{REFERENCES}

1. Agarwal, N. and Viniotis, I., "Performance Space of a GI/G/1 System Under a Percentile Goal Criterion," CCSP Report, North Carolina State University, Raleigh, NC, USA. 1993.

2. Bhattacharya, P.P. and Ephremides, E., "Optimal scheduling with strict deadlines," IEEE Trans. Automatic Control, Vol. 34, No. 7, pp. 721-728, Jul. 1989.

3. Federgruen, A. and Groenevelt, H., "M/G/C queueing systems with multiple customer classes: characterization and control of achievable performance under nonpreemptive priority rules", Management Science, Vol. 34, No. 9, pp. 1121-1138, 1988.

4. Gelenbe, E. and Mitrani, I.(1980). "Analysis and Synthesis of Computer Systems". Academic Press, New York.

5. Georgiadis, L. and Viniotis, I., "On the conservation laws and the performance space of single server systems", technical report RC 15673, IBM T.J. Watson Research Center, 1990.

6. Gopal, P.M. and Kadaba, B.K., "Network Delay Considerations for Packetized Voice," Performance Evaluation, Vol. 9, pp.167-180, 1988/89.

7. Kleinrock, L., "Queuing Systems Vol. I",. John Wiley \& Sons, New York, 1976.

8. Nagarajan, R. and Kurose, J., "On Defining, Computing, and Guaranteeing Qualityof-Service in High-Speed Networks," Proceedings of INFOCOM'92, pp.2016-2025, May 1992.

9. Nagarajan, R., Kurose, J., Towsley, D. and Hluchyj, M.G., "On per-session end-toend delay distributions and the call admission problem for real-time applications with QOS requirements", sigcomm, ACM, pp.2-12, Sep. 1993.

10. Takagi, Y., Hino, S. and Takahashi, T., "Priority Assignment Control of ATM Line Buffers with Multiple QOS Classes", IEEE Journal on Selected Areas in Communications, Vol. 9, No. 7, pp. 1078-1092, September 1991. 